\title{
PREVALENCIA DE FERROPENIA POR MENORRAGIA EN UNA INSTITUCIÓN EDUCATIVA DEL ÁREA DE LA SALUd EN COLOMBIA
}

\author{
Maria Helena Solano MD*, Irene Del Carmen González MD**
}

\section{Resumen}

La menorragia es la causa más frecuente de ferropenia en mujeres en edad reproductiva. Objetivo: describir la prevalencia de ferropenia en estudiantes del área de la salud mayores de 18 años y que no había sido detectada. Materiales y métodos: a 154 estudiantes de medicina (rango de edad: 18 a 36 años) se les aplicó el cuestionario PBAC (siglas en inglés de la tabla pictórica para la estimación del sangrado menstrual). Se describe la presencia de anemia ferropénica y los niveles de ferritina con frecuencias absolutas y relativas. Resultados: se detectó ferropenia en $15 \%$ (nivel sérico $<15 \mathrm{ug} / \mathrm{L}$ ), menorragia $3.25 \%$ (pérdida menstrual $>80 \mathrm{ml}$ por ciclo) y anemia en $\mathbf{2 \%}$, (hemoglobina $<12 \mathrm{~g} / \mathrm{dL}$ ). Conclusión: se observó alta prevalencia de ferropenia. Es necesario estudiar las causas con programas que incluyan promoción y evaluación de hábitos alimenticios en la población estudiantil. En nuestro estudio la herramienta no fue útil.

Palabras clave: menorragia, ferropenia, anemia, tabla pictórica para la estimación del sangrado menstrual (PBAC).

Abreviaturas: PBAC, tabla pictórica para la estimación del sangrado menstrual.

\section{PREVALENCE OF IRON DEFICIENCY DUE TO MENORRHAGIA IN MEDICAL STUDENTS IN COLOMBIA}

\begin{abstract}
Menorrhagia is the most common cause of iron deficiency in women of reproductive age. Objective: to describe the prevalence of iron deficiency, which had not been detected before, in older than 18 years old medical students. Materials and Methods: the PBAC (pictorial menstrual blood loss assessment chart) was applied to 154 medical students (aged: 18 to 36 years). The presence of iron-deficiency anemia and ferritin levels were described using absolute and relative frequencies. Results: iron deficiency was detected in $15 \%$ (serum level $<15 \mathrm{ug} / \mathrm{L}$ ), menorrhagia $3.25 \%$ (menstrual blood loss $>80 \mathrm{ml}$ per cycle) and anemia in $2 \%$, (hemoglobin $<12 \mathrm{~g} / \mathrm{dL})$. Conclusion: a high prevalence of iron deficiency was observed. A study on the causes by means of promotion programs and assessment of nutritional habits in the student population is needed. The tool used in our study was not useful.
\end{abstract}

Key words: menorrhagia, iron deficiency, anemia, pictorial menstrual blood loss assessment chart (PBAC)

Fecha recibido: julio 21 de 2014 - Fecha aceptado: marzo 10 de 2015

** Jefe del Servicio de Hematología, Hospital de San José, Profesor Titular, Fundación Universitaria de Ciencias de la Salud. Bogotá DC, Colombia.
* Residente IV de Hematología Hospital de San José. Bogotá DC, Colombia. 


\section{Introducción}

La ferropenia es uno de los motivos de consulta más frecuentes en la práctica de un hematólogo. En mujeres, de acuerdo con su peso corporal, las pérdidas de hierro basales pueden ser estimadas en $0.8 \mathrm{mg} /$ día y las menstruales de hierro deben cuantificarse con las pérdidas basales. ${ }^{1}$ De acuerdo con el estudio NHANES la prevalencia de anemia en mujeres jóvenes fue de $4.8 \%$, siendo la deficiencia de hierro la de mayor predominio. $^{2}$

Las mujeres requieren mayor cantidad de hierro que los hombres, además de sus necesidades comunes se enfrentan a demandas altas debido a la menstruación, el embarazo y la lactancia. Dentro de los factores que favorecen en las mujeres en edad reproductiva al desarrollo de ferropenia y anemia (forma grave de carencia de hierro) está la menorragia ${ }^{3}$, lo cual convierte a estas condiciones en un problema de salud pública por los síntomas asociados con la anemia, sobre el sistema inmunológico y a nivel neuromuscular ocasionando una reducción de la resistencia al ejercicio, de la actividad y de la productividad. ${ }^{4-6}$

Una de las pruebas para evaluar la ferropenia es la ferritina sérica, la cual tiene relación directa con los depósitos de hierro corporal $^{3}$ y es la prueba diagnóstica inicial preferida para detectar temprano la ferropenia. ${ }^{7}$ En edad reproductiva la sensibilidad de una concentración baja de ferritina sérica $(<15 \mu \mathrm{g} / \mathrm{L})$ para deficiencia de hierro es de $75 \%$ con una especificidad de $98 \%$. Cuando estas concentraciones son menores de $12 \mu \mathrm{g} / \mathrm{L}$ la sensibilidad para deficiencia de hierro es $61 \%$ con una especificidad de $100 \% .{ }^{8}$ Las manifestaciones de ferropenia en el cuadro hemático dependen de la severidad. Cuando la deficiencia de hierro es incipiente puede incluir solo anisocitosis leve, con aumento del ancho de distribución eritrocitaria (ADE, IDE o RDW); pero conforme se manifiesta la anemia (hemoglobina $<12 \mathrm{~g} / \mathrm{dL}$ ) aparecen hipocromía y microcitosis. El número de plaquetas puede estar dentro de lo normal, aunque cabe la posibilidad de trombocitopenia o trombocitosis.

De acuerdo con los estudios realizados por Haas y col. ${ }^{4}$ la ferropenia tiene consecuencias en el rendimiento laboral, calidad de vida y el desempeño intelectual; por lo anterior, es importante que los médicos que trabajan con esta población detecten en forma precoz la menorragia como un factor de riesgo para el desarrollo de deficiencia de hierro. Cuantificar la pérdida menstrual puede ser difícil en la práctica clínica. El promedio de duración de un sangrado menstrual normal es de cinco días con ciclos que varían de acuerdo con la edad, cuyo promedio de 28 a 30 días con una tendencia a acortarse cuando se incrementa la edad..$^{9,10}$

La menorragia se ha definido como una pérdida menstrual abundante pero regular, en la cual hay una pérdida de sangre mayor o igual a $80 \mathrm{ml}$ en cada menstruación. ${ }^{11,12}$ Se puede medir de tres formas: a) objetiva: cuantifica la hematina alcalina (del material sanitario usado se estima el contenido de sangre $)^{13}$; b) de acuerdo con los días de duración de la menstruación o el número de productos sanitarios utilizados $^{14}$ y c) la subjetiva o método de PBAC, de acuerdo con sus siglas en inglés (tabla pictórica para la estimación de sangrado menstrual), la cual se basa en la fracción de sangre que mancha el producto sanitario y el total de toallas o tampones utilizados. Un puntaje mayor de 100 es equivalente a una pérdida de sangre mayor de $80 \mathrm{ml}$. (Tabla 1). El PBAC tiene una especificidad de $89 \%$ y una sensibilidad de 86 $\% .^{15,16}$ Hay estudios que han demostrado como el incremento en las pérdidas sanguíneas menstruales altera los valores de ferritina sérica y hemoglobina, a partir de pérdidas menstruales de 60 a $80 \mathrm{ml}$ por ciclo (promedio de $76.4 \mathrm{ml})^{17}$, y la probabilidad de desarrollar anemia ferropénica se incrementa con pérdidas superiores a $120 \mathrm{ml}^{18}$

De acuerdo con los reportes de la OMS, la tasa de menorragia es de 8 a $27 \%$ en edad reproductiva basada en mediciones subjetivas. ${ }^{19}$ Estos resultados pueden reflejar las diferencias socioculturales en la percepción de la menstruación. La evidencia muestra que la menorragia tiene un efecto sobre la calidad de vida, con un impacto sobre la interacción social, bajo rendimiento y ausentismo laboral. ${ }^{14,20,21}$ En la práctica médica encontramos que menos de $50 \%$ consultan por pérdidas menstruales mayores de $80 \mathrm{ml}$ y casi un $50 \%$ que reportan menorragia tienen pérdidas menstruales menores de $40 \mathrm{ml}^{22,23}$ 


\section{Tabla I. Tabla pictórica para la estimación} semicuantitativa del sangrado menstrual

\begin{tabular}{|c|l|}
\hline Puntos & \multicolumn{1}{|c|}{ Interpretación } \\
\hline I & Por cada tampón vaginal con manchado ligero \\
\hline 5 & Por cada tampón vaginal con manchado moderado \\
\hline 10 & Por cada tampón vaginal completamente manchado \\
\hline I & Por cada compresa con ligera coloración \\
\hline 5 & Por cada compresa con moderada coloración \\
\hline 5 & $\begin{array}{l}\text { Por un gran coágulo. En caso de rebosamiento, se } \\
\text { estimará el valor aproximado. }\end{array}$ \\
\hline
\end{tabular}

Menorragia $=$ puntuación $\geq 100$. Bibliografía: Higham J, O' Brien PMS, Shaw RW. Assessment of menstrual blood loss using a pictorial chart. Br J Obstet Gynaecol 1990; 97: 734-39.

Se ha descrito anemia ferropénica en dos tercios de las mujeres con menorragia objetiva. ${ }^{10,24} \mathrm{Al}$ menos dos ensayos aleatorios controlados han demostrado la mejoría cognitiva y de la calidad de vida en las mujeres ferropénicas que reciben suplemento de hierro, aun en las que no se encontraban anémicas al momento del diagnóstico. ${ }^{25-27}$ El objetivo del artículo consiste en determinar la prevalencia de ferropenia por menorragia en un grupo de estudiantes de medicina mayores de 18 años y que no habían sido detectadas antes. Como objetivo específico se evaluó el estado de ferropenia y la presencia de anemia mediante ferritina sérica y hemograma.

\section{Materiales y métodos}

El diseño corresponde a un estudio de corte transversal. Se tomó como población a estudiantes mujeres de la facultad de medicina mayores de 18 años pertenecientes a una institución educativa en Colombia.

Se consideró como población objetivo todas las que aceptaron participar en forma voluntaria. Los criterios de exclusión fueron: a) embarazo en cualquier trimestre; b) patología neoplásica de cualquier sitio; y c) infección crónica, síndrome de malabsorción conocida o que se hubiera realizado algún tipo de cirugía bariátrica.

Luego de la aprobación por parte del comité de ética de la universidad, se invitó por semestres a las estudiantes y se explicaron los objetivos de la investigación y las que aceptaron participar se les entregó el consentimiento informado por escrito. Luego de aceptarlo se diligenció la escala subjetiva del ciclo menstrual PBAC y se realizaron las siguientes preguntas acerca de sus antecedentes personales:

a) ¿Sabe usted si sufre de anemia por deficiencia de hierro?

b) ¿Se encuentra en la actualidad en tratamiento con sulfato ferroso?

c) ¿Sabe si ha sufrido de anemia por deficiencia de hierro?

Después se procedió a la venopunción realizada por una flebotomista del laboratorio para la realización del hemograma y cuantificación de ferritina sérica. Dentro de los instrumentos de medición se contó con un formato de recolección de datos y los reportes de muestras biológicas tomadas por venopunción. Se creó una base datos en Microsoft Excel ${ }^{\circledR}$ y el análisis estadístico de la información se realizó en Stata versión 12.0 ®. Se reportó la prevalencia de deficiencia de hierro en porcentaje y la anemia ferropénica y los niveles de ferritina con frecuencias absolutas y relativas según las categorías establecidas.

\section{Resultados}

Se invitó a participar a todas las estudiantes de medicina de primero a décimo semestre mayores de 18 años, acudieron al llamado 154 (24\%) de un total de 629. El promedio de edad fue de 20.3 años (rango: 18 - 36). De las que participaron solo una tenía diagnóstico conocido de anemia ferropénica, quien no se encontraba recibiendo tratamiento con sulfato ferroso. Se detectó menorragia en $3.25 \%(\mathrm{n}=5)$, anemia $2 \%(n=3)$ y ferropenia en el $15 \%(n=22)$. De las 22 estudiantes en las que se detectó ferropenia, ninguna había sido diagnosticada antes y por lo tanto no venían recibiendo suplencia de hierro. Tres estudiantes presentaron anemia, dos de las cuales cumplieron con los criterios de cambios en la morfología del eritrocito: microcitosis, hipocromía y una cursó con trombocitosis asociada; ninguna fue detectada con menorragia. Las características clínicas y demográficas se resumen en la (Tabla 2). 


\begin{tabular}{|l|c|}
\hline \multicolumn{2}{|c|}{ Tabla 2. Características clínicas y demográficas } \\
\hline \multicolumn{1}{|c|}{ Caracteristicas clinicas, $\mathbf{n}$} & $\begin{array}{c}\text { Porcentaje } \\
(\mathbf{n})\end{array}$ \\
\hline Diagnóstico conocido de ferropenia & $0.1(\mathrm{I})$ \\
\hline Tratamiento con sulfato & 0 \\
\hline Ferropenia & $15(22)$ \\
\hline Anemia & $2(3)$ \\
\hline Hemoglobina corpuscular media & $1.9(3)$ \\
\hline Hematocrito & $1.3(2)$ \\
\hline Trombocitosis & $0.6(1)$ \\
\hline Menorragia & $3.2(5)$ \\
\hline
\end{tabular}

\section{Efectos de la escala}

La PBAC detectó cinco estudiantes con menorragia, de las cuales solo dos tenían ferropenia. En la (Figura 1) se relacionan las estudiantes con ferropenia y sus respectivos puntajes.

\section{Discusión}

Este es el primer estudio que evalúa la prevalencia de ferropenia por menorragia en estudiantes de medicina mayores de 18 años en Colombia. Se detectó menorragia en $3.25 \%$ una frecuencia baja de acuerdo con los datos de servicios de atención en salud, los cuales estiman que por lo menos de 5 a $10 \%$ de las mujeres en edad reproductiva buscan atención médica por menorragia. ${ }^{28,29}$ En este estudio no hubo ferropénicas con menorragia y las que referían menorragia no cursaron con ferropenia.

A pesar de conocer que la menstruación puede medirse en forma objetiva mediante la cuantificación de la hematina alcalina, en la que utilizando el material sanitario usado se estima el contenido de sangre, esto se hace sólo con fines de investigación y en los ensayos clínicos. Debido a que dos terceras partes de las mujeres con menorragia presentan anemia por deficiencia de hierro, se debe medir la hemoglobina y la ferritina sérica. ${ }^{30}$

La prevalencia de ferropenia fue de $15 \%$, lo que se considera como un porcentaje alto con respecto a otros estudios en este grupo etario; en otras investi- gaciones se ha descrito entre 10 y $11 \% .^{31,32}$ Existen publicaciones de deficiencia de hierro con frecuencias variables. Strain reporta una prevalencia de $11 \%$ en irlandesas. ${ }^{33}$ Switoniak $^{34}$ informa $13.4 \%$ en mujeres polacas, mientras que en Argentina se encontró $21.6 \%$ entre 16 y 44 años. ${ }^{35}$ Este último estudio asoció la ferropenia con el estado socioeconómico, encontrando que entre más bajo sea el nivel mayor será la frecuencia de ferropenia.

Se escogió como instrumento de medición la tabla PBAC teniendo en cuenta que existen al menos seis estudios que han evaluado la precisión diagnóstica en la detección de menorragia. El estudio de validación del instrumento desarrollado por Higham ${ }^{15}$ reportó una sensibilidad de $86 \%$ y especificidad $89 \%$, lo cual indica que es una herramienta muy válida que puede utilizarse en la práctica clínica. Un solo estudio no logró validar la tabla PBAC en una población con pérdidas menstruales sintomáticas obteniendo una sensibilidad de $97 \%$ y una especificidad de $7,5 \%{ }^{36}$, a diferencia de nuestro estudio cuya población era asintomática.

A pesar de que es una prueba fácil de realizar, en el estudio de Higham ${ }^{15}$ se reportaron mujeres con dificultad para entender la prueba, algunas anotaban menorragia en la tabla PBAC y resultaban con pérdidas menstruales normales, al parecer por dificultades para reconocer como es una toalla o

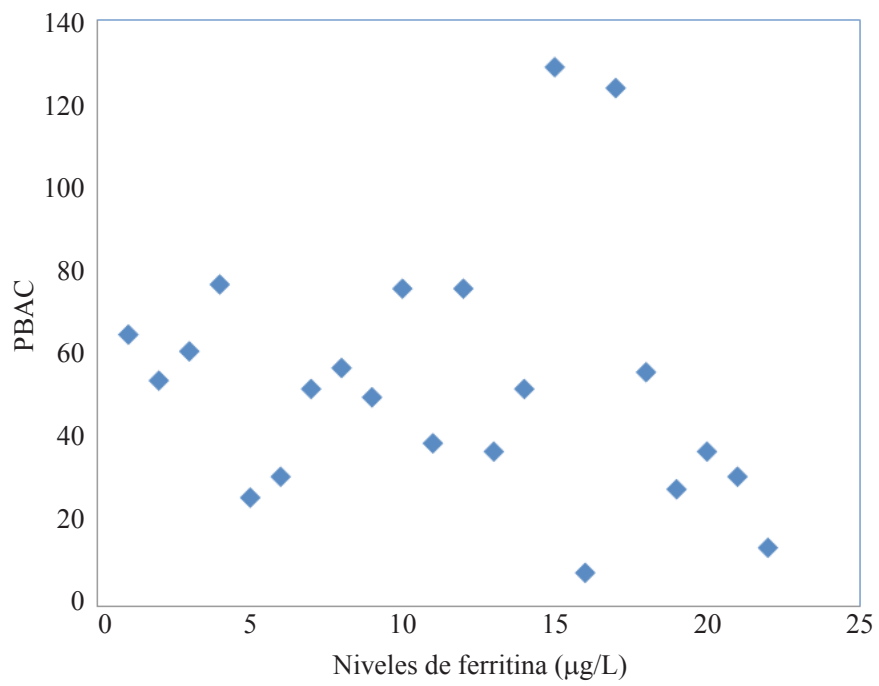

Figura I. Relación de niveles de ferropenia y puntaje PBAC. 
tampón con manchado leve a moderado. Otro factor que ha podido influir en los resultados es la diversidad en cuanto al tamaño y capacidad de absorción de los productos sanitarios, a diferencia con los estudios de menorragia en donde a las participantes se les suministran los productos sanitarios de la misma casa comercial.

En este estudio la frecuencia de anemia ferropénica fue de $2 \%$ (3 estudiantes) y ninguna cursó con menorragia, lo cual nos hace pensar que en nuestra población a estudio la ferropenia puede resultar de otras causas y no siempre refleja un trastorno ginecológico. Una de las razones que podrían plantearse es que en este grupo de edad, con el fin de evitar obesidad, siguen dietas autoformuladas, lo que podría llevar a desórdenes alimenticios y esta ser una de las razones por las que la ferropenia y la anemia ocurra en mujeres jóvenes ${ }^{37}$, pues la tendencia social y cultural es considerar la delgadez como ideal, lo cual afecta en especial a las adolescentes y mujeres jóvenes, lleva en algunos casos a una obsesión de reducir la ingesta de alimentos solo por razones estéticas. ${ }^{35}$ Así, la identificación de la ferropenia es importante para estratificar a esas estudiantes con etiología no clara, en búsqueda de otras causas. De acuerdo con los resultados de los estudios mencionados antes, asumimos que iniciar la suplencia de hierro en las estudiantes detectadas con ferropenia, podría evitar el desarrollo de anemia con las consecuencias ya descritas. No existen estudios de nuestro país en donde se haya evaluado la prevalencia de ferropenia por menorragia en este grupo de edad.

La presencia de ferropenia y anemia se asocia en forma significativa con una reducción en la calidad de vida. ${ }^{20}$ En estudios futuros es importante valorarla así como la función cognitiva con el fin de describir la disminución en ambas áreas. La principal limitación fue la baja participación por parte de las estudiantes, una de las causas fue el temor a la venopunción y motivos personales asociados con el entorno social generado por sus compañeros de curso. Estos antecedentes demuestran la necesidad de aplicar otros métodos o herramientas que permitan detectar la presencia de menorragia de una manera menos subjetiva.

\section{Conclusiones}

En nuestra población se presentó alta prevalencia de ferropenia y se hace necesario estudiar las causas por medio de programas que incluyan la promoción y la evaluación de hábitos alimenticios en la población estudiantil. En cuanto a la herramienta, no es útil en una población joven asintomática como es el caso de las estudiantes de medicina.

\section{Referencias}

1. Hercberg S, Galan P. Nutritional anaemias. Baillieres Clin Haematol. 1992; 5(1):143-68.

2. Dallman PR, Yip R, Johnson C. Prevalence and causes of anemia in the United States, 1976 to 1980. Am J Clin Nutr. 1984; 39(3):437-45.

3. Recommendations to prevent and control iron deficiency in the United States. Centers for Disease Control and Prevention. MMWR Recomm Rep. 1998; 47(RR-3):1-29.

4. Haas JD, Brownlie T. Iron deficiency and reduced work capacity: a critical review of the research to determine a causal relationship. J Nutr. 2001; 131(2S-2):676S88S; discussion 88S-90S.

5. Edgerton VR, Gardner GW, Ohira Y, Gunawardena KA, Senewiratne B. Irondeficiency anaemia and its effect on worker productivity and activity patterns. $\mathrm{Br}$ Med J. 1979; 2(6204):1546-9.

6. Brock JH, Mulero V. Cellular and molecular aspects of iron and immune function. Proc Nutr Soc. 2000; 59(4):537-40.

7. Patterson AJ, Brown WJ, Roberts DC. Dietary and supplement treatment of iron deficiency results in improvements in general health and fatigue in Australian women of childbearing age. J Am Coll Nutr. 2001; 20(4):337-42.

8. Murray Kolb LE. Iron status and neuropsychological consequences in women of reproductive age: what do we know and where are we headed?. J Nutr. 2011; 141(4):747S-55S.

9. Münster K, Schmidt L, Helm P. Length and variation in the menstrual cycle-a cross-sectional study from a Danish county. Br J Obstet Gynaecol. 1992; 99(5):422-9.

10. Chiazze L, Brayer FT, Macisco JJ, Parker MP, Duffy BJ. The length and variability of the human menstrual cycle. JAMA. 1968; 203(6):377-80.

11. Hallberg L, Nilsson L. Determination of menstrual blood loss. Scand J Clin Lab Invest. 1964; 16:244-8.

12. Fraser IS, Warner P, Marantos PA. Estimating menstrual blood loss in women with normal and excessive menstrual fluid volume. Obstet Gynecol. 2001; 98(5 Pt 1):806-14.

13. Janssen CA, Scholten PC, Heintz AP. A simple visual assessment technique to discriminate between menorrhagia and normal menstrual blood loss. Obstet Gynecol. 1995; 85(6):977-82.

14. Warner PE, Critchley HO, Lumsden MA, Campbell-Brown M, Douglas A, Murray GD. Menorrhagia II: is the $80-\mathrm{mL}$ blood loss criterion useful in management of complaint of menorrhagia?. Am J Obstet Gynecol. 2004; 190(5):1224-9.

15. Higham JM, O'Brien PM, Shaw RW. Assessment of menstrual blood loss using a pictorial chart. Br J Obstet Gynaecol. 1990; 97(8):734-9.

16. Wyatt KM, Dimmock PW, Walker TJ, O'Brien PM. Determination of total menstrual blood loss. Fertil Steril. 2001; 76(1):125-31.

17. Hallberg L, Högdahl AM, Nilsson L, Rybo G. Menstrual blood loss--a population study. Variation at different ages and attempts to define normality. Acta Obstet Gynecol Scand. 1966; 45(3):320-51. 
18. Janssen CA, Scholten PC, Heintz AP. Reconsidering menorrhagia in gynecological practice. Is a 30-year-old definition still valid?. Eur J Obstet Gynecol Reprod Biol. 1998; 78(1):69-72.

19. Harlow SD, Campbell OM. Epidemiology of menstrual disorders in developing countries: a systematic review. BJOG. 2004; 111(1):6-16.

20. Shapley M, Jordan K, Croft PR. Increased vaginal bleeding: the reasons women give for consulting primary care. J Obstet Gynaecol. 2003; 23(1):48-50.

21. Barnard K, Frayne SM, Skinner KM, Sullivan LM. Health status among women with menstrual symptoms. J Womens Health (Larchmt). 2003; 12(9):911-9.

22. Jenkinson C, Peto V, Coulter A. Making sense of ambiguity: evaluation in internal reliability and face validity of the SF 36 questionnaire in women presenting with menorrhagia. Qual Health Care. 1996; 5(1):9-12.

23. Von Mackensen S. Quality of life in women with bleeding disorders. Haemophilia. 2011; 17 Suppl 1:33-7.

24. Clark TJ, Khan KS, Foon R, Pattison H, Bryan S, Gupta JK. Quality of life instruments in studies of menorrhagia: a systematic review. Eur J Obstet Gynecol Reprod Biol. 2002; 104(2):96-104.

25. Youdim MB, Yehuda S. The neurochemical basis of cognitive deficits induced by brain iron deficiency: involvement of dopamine-opiate system. Cell Mol Biol (Noisy-le-grand). 2000; 46(3):491-500.

26. Coulter A, Peto V, Jenkinson C. Quality of life and patient satisfaction following treatment for menorrhagia. Fam Pract. 1994; 11(4):394-401.

27. Bruner AB, Joffe A, Duggan AK, Casella JF, Brandt J. Randomised study of cog nitive effects of iron supplementation in non-anaemic iron-deficient adolescent girls. Lancet. 1996; 348(9033):992-6.
28. Hallberg L, Bengtsson C, Lapidus L, Lindstedt G, Lundberg PA, Hultén L. Screening for iron deficiency: an analysis based on bone-marrow examinations and serum ferritin determinations in a population sample of women. Br J Haematol. 1993; 85(4):787-98.

29. Warner PE, Critchley HO, Lumsden MA, Campbell-Brown M, Douglas A, Murray GD. Menorrhagia I: measured blood loss, clinical features, and outcome in women with heavy periods: a survey with follow-up data. Am J Obstet Gynecol. 2004; 190(5):1216-23.

30. Vessey MP, Villard-Mackintosh L, McPherson K, Coulter A, Yeates D. The epidemiology of hysterectomy: findings in a large cohort study. Br J Obstet Gynaecol. 1992; 99(5):402-7.

31. Oehler MK, Rees MC. Menorrhagia: an update. Acta Obstet Gynecol Scand. 2003; 82(5):405-22.

32. Management of menorrhagia. BMJ. 1990; 301(6746):290-1.

33. Strain JJ, Thompson KA, Barker ME, Carville DG. Iron sufficiency in the population of Northern Ireland: estimates from blood measurements. Br J Nutr. 1990; 64(1):219-24.

34. Switoniak T, Król A. [Iron deficiency and anemia in professional working women]. Przegl Epidemiol. 1992; 46(4):379-87.

35. Calvo EB, Sosa EM. Iron status in non-pregnant women of child-bearing age living at Greater Buenos Aires. Eur J Clin Nutr. 1991; 45(4):215-20.

36. Reid PC, Coker A, Coltart R. Assessment of menstrual blood loss using a pictorial chart:a validation study. BJOG. 2000;107(3):320-2.

37. Bothwell TH, Charlton RW. Iron deficiency in women. Washington, D.C: In ternational Nutritional Anemia Consultative Group; 1981 Apr. Document N.: 052386. Sponsored by the Nutrition Foundation. 